\title{
Critical Behavior of High Temperature Superconductors
}

\author{
Claude de Calan \\ Centre de Physique Théorique, \\ Ecole Polytechnique, 91128 PALAISEAU CEDEX, France \\ E-mail : calan@cpht.polytechnique.fr
}

Received on 5 January, 2001

\begin{abstract}
We consider the scaling behavior in the critical domain of superconductors at zero external magnetic field. The Josephson's relation for a charged superfluid is proved without assuming the hyperscaling relation. On the other hand we discuss the dual Ginzburg-Landau model. In this dual model, due to the presence of two mass scales, a continuous family of non equivalent scalings can be defined. The relevant critical regimes are identified, and the corresponding critical exponents are predicted.
\end{abstract}

\section{Introduction}

The study of high temperature superconductors (HTSC's) is not an easy one, from a theoretical point of view as well as from an experimental one.

Up to now, there is no theory of the microscopic mechanism generating the pairing of electrons which leads to superconductivity, for these HTSC's. Therefore the theoretical study can be performed only in the frame of phenomenological models, the most popular of which is the Ginzburg-Landau (GL) model. But the question raises : is this GL model relevant for HTSC's, at least for what concerns the critical behavior, in the neighborhood of the superconducting transition ? In other words, do the HTSC's and the GL model belong to the same universality class? As many people believe, we claim that it is the case, but this can be checked only by comparison with experiments.

Now the experimental situation is also a bit confusing[1]. Different experiments, performed on different materials, give different results. Many effects have to be taken into account :

i) finite size effects (in particular in one of the three space dimensions, for thin films samples) ;

ii) anisotropy effects, since the HTSC's generally present a crystalline structure made of bidimensional layers ;

iii) estimate of the critical region probed, depending on how much the critical temperature is approached.

We shall be concerned here with the critical behavior of HTSC's at zero external magnetic field, and we are mainly interested in the values of the critical exponents $\nu$ (the exponent for the inverse of $\xi$, where $\xi$ is the correlation length) and $\nu^{\prime}$ (the exponent for the inverse of $\lambda$, where $\lambda$ is the penetration depth). The experimental results, for approximately isotropic, extreme type II superconductors are essentially the following ones :

Almost all experimentalists agree on the value of $\nu$, approximately 0.67 , but some find $\nu^{\prime}=\nu / 2 \simeq 0.33$. This corresponds to an uncharged three-dimensional $\mathrm{XY}$ universality class, which must be relevant for a critical region near, but not very near, from the critical temperature ; a region where the gauge field fluctuations can be neglected.

Some other experimentalists, working with thin films, find $\nu \simeq 0.67$ and $\nu^{\prime}=1 / 2$. In this case, the value of $\nu^{\prime}$ corresponds to a mean-field-like behavior.

We stress that the charged critical region, very near of the critical temperature, is a very small region, not yet accessible to the experimental probes. And we shall see that in the charged regime the critical exponents must take the values $\nu=\nu^{\prime} \simeq 0.67$. This prediction is confirmed by a recent numerical study on the lattice[2].

In section II, we set up the main features we get from the renormalization group study of the GL model. Section III gives exact non perturbative relations between critical exponents, including a proof of Josephson's relation and of hyperscaling. In section IV, we consider the dual GL model and we elucidate the controversy which happened among theoreticians, depending on the definition of the scaling.

\section{The Ginzburg-Landau model}

The action for the GL model is built from the minimal electromagnetic coupling of the Abelian gauge field with a charged scalar field, and an additional quartic 
self-coupling of the scalar field :

$$
\begin{gathered}
S=\int d^{3} x\left\{\frac{1}{2}\left(\vec{\nabla} \wedge \overrightarrow{A_{0}}\right)^{2}+\left|\left(\vec{\nabla}-i e_{0} \overrightarrow{A_{0}}\right) \phi_{0}\right|^{2}\right. \\
\left.+m_{0}^{2}\left|\phi_{0}\right|^{2}+\frac{u_{0}}{2}\left|\phi_{0}\right|^{4}\right\}
\end{gathered}
$$

$\overrightarrow{A_{0}}$ is the electromagnetic field

$\Phi_{0}$ is the bare order parameter (related to electron's pairs)

$m_{0}$, the bare mass of $\phi_{0}$, is related to the temperature by

$$
m_{0}^{2}=T-T_{c}=t
$$

The renormalized quantities $\vec{A}, \phi, m, u, e$ are defined in the standard way :

$$
\begin{gathered}
\overrightarrow{A_{0}}=Z_{A}^{\frac{1}{2}} \vec{A} \\
\phi_{0}=Z_{\phi}^{\frac{1}{2}} \phi \\
m_{0}^{2}=Z_{\phi}^{(2)} Z_{\phi}^{-1} m^{2} \\
u_{0}=Z_{u} Z_{\phi}^{-2} u \\
e_{0}=Z_{A}^{-\frac{1}{2}} e
\end{gathered}
$$

From gauge invariance, the same factor $Z_{A}$ enters the definition of $\vec{A}$ and $e$. The renormalized mass $m$ is the inverse of the correlation length $\xi: m=\xi^{-1}$ and the critical exponent $\nu$ is defined by $m \sim t^{\nu}$ when $t \rightarrow 0$.

In dimension 3 , we define the dimensionless couplings $f$ and $v$ by

$$
f=e^{2} / m \quad \text { and } \quad v=u / m
$$

The renormalization group equations are established by choosing $m$ as the scaling parameter and differentiating with respect to $\ln m$, at fixed $u_{0}$ and $e_{0}$.

Working at fixed dimension $d=3$ in the one-loop approximation, one finds the flow diagram[3]:

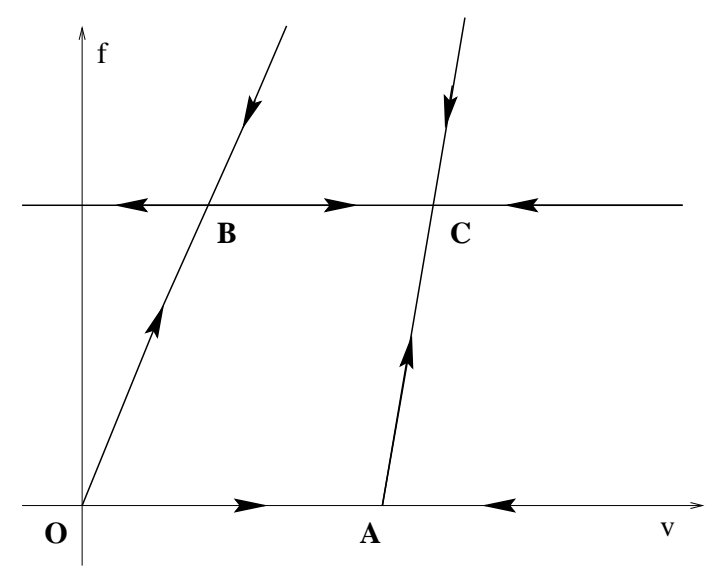

Figure 1
In Fig.1, the arrows give the evolution of $(v, f)$ when $m$ goes to zero. Four fixed points appear:

$\mathrm{O}$ is the trivial Gaussian fixed point, infrared repulsive.

A is the neutral $(e=0) \mathrm{XY}$ point, infrared attractive only at zero charge (i.e. in the $\phi^{4}$ model).

$\mathrm{B}$ is a tricritical point. The line from A to B separates two basins : starting on the left of this line, $v$ goes to negative values and there is no fixed point, which corresponds to a first order transition ; starting on the right, $(v, f)$ goes to the attractive, IR stable, charged fixed point $\mathrm{C}$, which corresponds to a second order transition.

It must be noticed that when working with the traditional $\epsilon$ expansion, where $\epsilon=4-d$, the charged fixed points $\mathrm{B}$ and $\mathrm{C}$ are not found[4], unless $\phi$ is an Ncomponent field with a very large number of components $(\mathrm{N}>365)$. The absence of charged fixed points corresponds to a first-order transition, appropriate for the description of superconductors in the type I regime, but not for the type II regime.

The $4-\epsilon$ method provides a good control of the renormalization group, as far as one is interested in a dimension equal to (or near from) 4. But for $\epsilon=1$ (in dimension 3), this control is usually lost. On the other hand, the control in our method (fixed $d=3$ ) depends on the smallness of the coupling constants. At the charged fixed point, these constants are not really small. However one may hope that the one-loop contributions remain dominant, as it generally happens. Furthermore, we shall see in section 4 below, from the dual GL model, a qualitative confirmation of the results.

\section{Exact non perturbative re- sults}

In this section we take an arbitrary (yet fixed) dimension $d, 2<d \leq 4$, and we define correspondingly :

$$
\begin{aligned}
& f=e^{2} m^{d-4} \\
& v=u m^{d-4}
\end{aligned}
$$

We shall prove some exact results (not depending on the one-loop approximation), with only one assumption, the existence of the stable infrared fixed point[5].

The $\beta$-functions for the renormalization constants are defined as :

$$
\begin{aligned}
\eta_{A} & =m \frac{\partial}{\partial m} \ln Z_{A} \\
\eta_{\phi} & =m \frac{\partial}{\partial m} \ln Z_{\phi}
\end{aligned}
$$




$$
\eta_{\phi}^{(2)}=m \frac{\partial}{\partial m} \ln Z_{\phi}^{(2)}
$$

$Z_{A}$ is defined from the two-points function for the $\vec{A}$ field. At the infrared fixed point, $\eta_{A} \rightarrow \eta_{A}^{*}$ when $m \rightarrow 0, \eta_{A}^{*}$ being the anomalous dimension of the $\vec{A}$ field.

Similarly, $Z_{\phi}$ is defined from the two-points function of the $\phi$ field, and $Z_{\phi}^{(2)}$ from the two-points function of $\phi$ with mass insertion. At the infrared fixed point, $\eta_{\phi} \rightarrow \eta_{\phi}^{*}=\eta, \eta$ being the anomalous dimension of $\phi$.

From the definition of $f$, its $\beta$-function is :

$$
m \frac{\partial}{\partial m} f=\left(\eta_{A}+d-4\right) f
$$

Assuming $f \rightarrow f^{*} \neq 0$ when $m \rightarrow 0$, since $m \frac{\partial}{\partial m} f$ must vanish at the fixed point, we find :

$$
\eta_{A}^{*}=4-d
$$

Now in the normal phase $\left(m_{0}^{2}>0, T>T_{c}\right)$ the photon is massless. But in the Meissner phase $\left(m_{0}^{2}<\right.$ $0, T<T_{c}$ ), it is well known that the symmetry is broken, the classical potential becomes a double-well one and the photon acquires a mass $m_{A}$. The critical exponent of $m_{A}$ is labeled as $\nu^{\prime}$ :

$$
m_{A} \sim t^{\nu^{\prime}}
$$

and its inverse $\lambda=m_{A}^{-1}$ is the penetration depth.

The mass $m_{A}$ is given by

$$
m_{A}^{2}=e^{2} \rho_{s}
$$

where the superfluid density $\rho_{s}=<|\phi|^{2}>$ satisfies

$$
\rho_{s}=\frac{m^{2}}{u}
$$

The Ginzburg parameter $\kappa$ is defined as the ratio of the two masses. Thus we have :

$$
\kappa=\frac{m}{m_{A}}=\left(\frac{u}{e^{2}}\right)^{\frac{1}{2}}=\left(\frac{v}{f}\right)^{\frac{1}{2}}
$$

From the definition of $m_{A}$, the $\beta$-function for $m_{A}^{2}$ is :

$$
m \frac{\partial}{\partial m} m_{A}^{2}=\left(\eta_{A}+d-2-\frac{1}{v} m \frac{\partial}{\partial m} v\right) m_{A}^{2}
$$

At the infrared fixed point, $v \rightarrow v^{*} \neq 0, m \frac{\partial}{\partial m}$ vanishes and $\eta_{A} \rightarrow \eta_{A}^{*}=4-d$. Therefore

$$
m \frac{\partial}{\partial m} m_{\geq}^{2} \rightarrow 2 m_{A}^{2}
$$

This means that $m_{A}$ behaves like $m$, and we find :

$$
\nu=\nu^{\prime}
$$

Remembering $m_{A}^{2}=e^{2} \rho_{s}$ and using $\nu=\nu^{\prime}$, we find for the critical behavior of $\rho_{s}$ :

$$
\rho_{s} \sim t^{\nu(d-2)}
$$

which is nothing but the Josephson's relation[6]. In contrast with what is usually done, we prove this relation without using the hyperscaling relation.

On the other hand

$$
\rho_{s}=<|\phi|^{2}>=Z_{\phi}^{-1}<\left|\phi_{0}\right|^{2}>
$$

Calling $\beta$ the critical exponent of $\phi_{0}$ and remembering $Z_{\phi} \sim m^{\eta}$, we can write the critical exponent of $\rho_{s}$ as $2 \beta-\nu \eta$. But the relation $2 \beta-\nu \eta=\nu(d-2)$ has been shown[7] to hold only if the hyperscaling relation $d \nu=2-\alpha$ holds ( $\alpha$ being the critical exponent for the specific heat. Therefore we also prove the hyperscaling relation for the GL model.

Finally we may notice that if the gauge field fluctuations were neglected, i.e. at zero charge, we would have $\eta_{A}^{*}=0$ (instead of $4-d$ ) and consequently $m_{A}^{2} \sim m^{d-2}$ (instead of $\mathrm{m}^{2}$ ). Therefore in this case

$$
\nu^{\prime}=\frac{\nu(d-2)}{2}
$$

and $\nu^{\prime}=\frac{\nu}{2}$ for $d=3$. We recover the XY behavior which corresponds to approaching the neutral fixed point A (see fig. 1).

\section{The dual Ginzburg-Landau model}

The dual GL model has been proposed using plausible arguments on the dynamics of a vortex gas[8]. In principle, it is the continuum limit of the geometrical dual (on the lattice) of the lattice version for the direct GL model. Lattice duality in Abelian gauge models have been used to predict that a second order phase transition should take place, at least in the type II regime[9].

But the continuous limit giving the continuum version of the dual GL model is not easy to perform, and implies several approximations. We do not repeat here the arguments leading to this dual model, which are set up in our paper[5]. The result is the following action :

$$
\begin{gathered}
S=\int d^{3} x\left\{\frac{1}{2}\left(\vec{\nabla} \wedge \overrightarrow{h_{0}}\right)^{2}+\frac{1}{2} M_{0}^{2}{\overrightarrow{h_{0}}}^{2}+\left|\left(\vec{\nabla}-i M_{0} q_{0} \overrightarrow{h_{0}}\right) \psi_{0}\right|^{2}\right. \\
\left.+\mu_{0}^{2}\left|\psi_{0}\right|^{2}+\frac{w_{0}}{2}\left|\psi_{0}\right|^{4}\right\}
\end{gathered}
$$

The dual GL model is thus analogous to the direct GL model, but the bare gauge field $\vec{h}_{0}$ (related to the magnetic induction) is now a massive field, with mass $M_{0}$. 
This is similar to what happens in the broken symmetry region of the direct model. Thus the duality exchanges the ordered and disordered phases, and the charged scalar field $\psi_{0}$ is sometimes called a "disorder parameter". On the other hand, the bare charge $q_{0}$ is related to the bare charge $e_{0}$ of the direct model by the Dirac's relation

$$
q_{0}=\frac{2 \pi}{e_{0}}
$$

Thus the duality also exchanges the large and small coupling situations, giving a better support to the small coupling expansion implied in our (fixed dimension) method.

The renormalized quantities in the dual model are defined in a way quite analogous to the previous one :

$$
\begin{gathered}
\vec{h}_{0}=Z_{h}^{\frac{1}{2}} \vec{h} \\
\psi_{0}=Z_{\psi}^{\frac{1}{2}} \psi \\
\mu_{0}^{2}=Z_{\psi}^{(2)} Z_{\psi}^{-1} \mu^{2} \\
M_{0} q_{0}=Z_{h}^{-\frac{1}{2}} M q \\
w_{0}=Z_{w} Z_{\psi}^{-2} w
\end{gathered}
$$

After renormalization, one has to identify the critical behavior of $\mu$ and $M$ with the critical behavior of $m$ and $m_{A} *$ in the direct model, retaining the same critical exponents $\nu$ and $\nu^{\prime}$

$$
\mu \sim t^{\nu}, \quad M \sim t^{\nu^{\prime}}
$$

In particular, the dual Ginzburg parameter

$$
\kappa_{d}=\frac{\mu}{M}
$$

has the same fixed point value as the direct Ginzburg parameter

$$
\kappa=\frac{m}{m_{A}}
$$

Now a problem comes from the fact that, from the beginning, two masses $M_{0}$ and $\mu_{0}$ enter the action of the dual model : taking $\mu$ as the scaling parameter, what is the scaling for $M_{0}$ ? In principle, the scaling law for $M_{0}$ must be deduced from the relation between the direct and the dual GL models. But since the steps leading to the dual model are not simple, this relation is practically lost. By working just in the dual model (forgetting where it comes from), one has to assume the $M_{0}$ scaling behavior. Indeed, various authors made different assumptions, which led to some controversy. Let us generally define the $M_{0}$ scaling behavior by

$$
M_{0}^{2} \sim t^{\zeta}
$$

and look at the results of the renormalization group study.

i) If $\zeta$ is taken to be zero, it amounts to take the limit $\mu \rightarrow 0$ at a fixed value of $M_{0}$. The result is then[10]

$$
\nu^{\prime}=\frac{\nu}{2}
$$

with a vanishing limit for the Ginzburg parameter $\kappa$. This is characteristic of a neutral XY behavior (the only one presently seen by the experimentalists) and is related to the approach of the neutral fixed point $\mathrm{A}$ in the direct model.

ii) If $\zeta$ is taken to be 1 , it amounts to identify $M_{0}$ with the bare mass $m_{A_{0}}$ of the gauge field in the broken symmetry phase of the direct model. The result here becomes[11]

$$
\nu^{\prime}=\frac{1}{2}
$$

a mean-field value for the exponent of the penetration depth. As we saw in the introduction, this could be a relevant behavior for the superconducting transition of thin films.

iii) If and only if we take $\zeta=2 \nu$, which amounts to identify the scaling behavior of $M_{0}$ with the scaling behavior of the renormalized mass $m_{A}$, we find[5]

$$
\nu=\nu^{\prime}
$$

with a non-vanishing fixed-point value of the Ginzburg parameter. This choice now corresponds to the charged regime, approaching the infrared stable fixed point $\mathrm{C}$ of the direct model.

At the end of this short review, let us mention that it would be very useful to ask for the same questions in presence of an external magnetic field. Many recent experiments are concerned with such a situation, and indicate that several transitions occur, between various phases. Starting from the normal metallic phase, when the temperature is decreasing (depending on the value of the external field), one probably meets a phase with a fluid of vortices, then the Abrikosov's phase with a regular lattice of vortices, before reaching the Meissner superconducting phase.

But from a theoretical point of view the problem is extremely hard. We are presently working on this subject, trying to go farther than the old results of ref.[12]. 


\section{References}

[1] M.B. Salamon, J. Shi, N. Overend and M.A. Howson, Phys. Rev. B 47, 5520 (1993); N. Overend, M.A. Howson and I.D. Lawrie, Phys. Rev. Lett. 72, 3238 (1994); S. Kamal, D.A. Bonn, N. Goldenfeld, P.J. Hirschfeld, R. Liang and W.N. Hardy, Phys. Rev. Lett. 73, 1845 (1994); S. Kamal, R. Liang, A. Hosseini, D.A. Bonn and W.N. Hardy, Phys. Rev. B 58, R8933 (1998); K.M. Paget, B.R. Boyce and T.R. Lemberger, Phys. Rev. B 59, 6545 (1999); Z.H. Lin, G.C. Spalding, A.M. Goldman, B.F. Bayman and O.T. Valls, Europhys. Lett. 32, 573 (1995).

[2] P. Olsson and S. Teitel, Phys. Rev. Lett. 80, 1964 (1998).

[3] I.F. Herbut and Z. Tesanovic, Phys. Rev. Lett. 76, 4588 (1996) ; I.D. Lawrie, Phys. Rev. Lett. 78, 979 (1997) ; I.F. Herbut and Z. Tesanovic, Phys. Rev. Lett. 78, 980 (1997) ; C. de Calan, A.P.C. Malbouisson, F.S. Nogueira and N.F. Svaiter, Phys. Rev. B 59, 554 (1999).

[4] B.I. Halperin, T.C. Lubensky and S.K. Ma, Phys. Rev. Lett. 32, 292 (1974) ; J.H. Chen, T.C. Lubensky and D.R. Nelson, Phys. Rev. B 17, 4274 (1978).
[5] C. de Calan and F.S. Nogueira, Phys. Rev. B 60, 4255 (1999).

[6] B.D. Josephson, Phys. Lett. 21, 608 (1966).

[7] M.E. Fisher, M.N. Barber and D. Jasnow, Phys. Rev. A 8, 1111 (1973).

[8] M. Kiometzis, H. Kleinert and A.M.J. Schakel, Phys. Rev. Lett. 73, 1975 (1994) ; A. Kovner, P. Kurzepa and B. Rosenstein, Mod. Phys. Lett. A 8, 1343 (1993) ; M. Kiometzis, H. Kleinert and A.M.J. Schakel, Fortschr. Phys. 43, 697 (1995) and references therein.

[9] C. Dasgupta and B.I. Halperin, Phys. Rev. Lett. 47, 1556 (1981) ; M.E. Peskin, Ann. Phys. (N.Y.) 113, 122 (1978) ; P.R. Thomas and M. Stone, Nucl. Phys. B 144, 513 (1978).

[10] I.F. Herbut, J. Phys. A 30, 423 (1997).

[11] M. Kiometzis, H. Kleinert and A.M.J. Schakel, Phys. Rev. Lett. 73, 1975 (1994)

[12] E. Brezin, D.R. Nelson and A. Thiaville, Phys. Rev. B 31, 7124 (1985). 